LAWRENCE LIVERMORE N A T IO N A L LABORATORY

Field Analysis of Propellant Stabilizers and their Daughter Products in any Propellant Formulation by Thin-Layer Chromatography Year End Report 2003

R. Whipple

December 9, 2003 
This document was prepared as an account of work sponsored by an agency of the United States Government. Neither the United States Government nor the University of California nor any of their employees, makes any warranty, express or implied, or assumes any legal liability or responsibility for the accuracy, completeness, or usefulness of any information, apparatus, product, or process disclosed, or represents that its use would not infringe privately owned rights. Reference herein to any specific commercial product, process, or service by trade name, trademark, manufacturer, or otherwise, does not necessarily constitute or imply its endorsement, recommendation, or favoring by the United States Government or the University of California. The views and opinions of authors expressed herein do not necessarily state or reflect those of the United States Government or the University of California, and shall not be used for advertising or product endorsement purposes.

This work was performed under the auspices of the U.S. Department of Energy by University of California, Lawrence Livermore National Laboratory under Contract W-7405-Eng-48. 
UCRL 303212

\title{
Field Analysis of Propellant Stabilizers and Their Daughter Products in Any Propellant Formulation by Thin-Layer Chromatography Year End Report 2003
}

\author{
Principal Investigator: $\quad$ Richard E. Whipple \\ Phone: $\quad 925-422-6314$ \\ FAX: $\quad$ 925-423-9014 \\ Email: $\quad$ whipple2@1lnl.gov
}
Address: $\quad$ Lawrence Livermore National Laboratory
7000 East Ave. L-178
Livermore, CA 94550

Project Team: Greg Klunder, Jeanne Bazan, Pete Nunes, Marina Chiarappa-Zucca

\section{Executive Summary:}

Lawrence Livermore National Laboratory’s Forensic Science Center (FSC) has successfully resolved a variety of concerns related to propellant stabilizer analysis by thin-layer chromatography. The technology is now ready to proceed to validation and the related issues associated with deployment, both CONUS and OCONUS.

\section{Year-End Project Summary:}

The goal of this project is to develop a completely self-contained field-portable system to quantitatively analyze propellants for their stabilizer content. Lawrence Livermore National Laboratory's Forensic Science Center (FSC) has taken an established technology, thin-layer chromatography (TLC), and developed new sampling protocols, unique hardware, and specialized chemistries for incorporation into a new field-portable TLC kit. Some of the advantages of this system are the ability to analyze several samples or lots simultaneously, very low detection limits, and field reliability when deployed, it will become an efficient, costeffective method for the determination of the remaining effective stabilizer (\%RES) in propellants. Due to its portability, ease of use, and low cost, this technology will be useful in a variety of CONUS and OCONUS analysis scenarios. The TLC technology development is sponsored by the US Army, Defense Ammunition Center (DAC). Patents on this technology have been awarded and the kit is now commercially available. 
Propellant analysis using the portable TLC kit has been demonstrated at several venues over the last few years. Initial results obtained at the FSC and in the field were consistent with values obtained by high-performance liquid chromatography (HPLC) at the U.S. Army Armament, Research, Development and Engineering Center (ARDEC). However, a unique problem began to develop that affected the results in a number of inconsistent ways. In an attempt to solve this problem, a joint meeting with ARDEC, Geo-Centers, the Defense Ammunition Center, and FSC staff was conducted last fall. From that meeting, a plan was developed to address the issues related to those inconsistencies and the necessary steps were designed for progression to validation. Although the main difficulty appeared to be related to humidity, other areas such as TLC plate variability, the digital imaging system, and the chemical reagents were also suspect. To better delineate the observed variation between the lab and the field, a collaborative effort with Geo-Centers was outlined. Using the FSC's TLC system, the two labs addressed the general inconsistencies as well as the effects of ambient temperature and humidity.

Humidity was the initially variable of concern although after extensive testing the TLC plates became the focus. The commercial TLC plates were different from those used previously in the project. The manufacturer's product number had been constant throughout this project, but analyte spot resolution and the resulting analysis had become inconsistent when compared to data from analyses conducted in 2000 . Until this plate variability question could be resolved, all work at Geo-Centers was stopped. Upon discussing the TLC plate problems with the manufacturer, Macherey-Nagel GmbH \& Co. in Germany it was disclosed that, although the product number has been the same throughout, the materials used to fabricate the plates had changed somewhat. The input materials used in 1999 and those used from that time through the fall of 2002, were not the same and likely resulted in the resolution irregularities. We were also informed that the current input materials had been readjusted to again be the same as those used in 1999. Upon testing at the FSC, the new plates gave results identical to or better than those achieved with plates manufactured in 1999. The three figures on page 3 show TLC chromatographs obtained with the original plates used on the project in 1999, those used until early 2003, and those in use now. Note the separations between spots or resolution. Images \#1 and \# 2 are from propellant interrogations in November of 2000 and March of 2002, respectively. Image \#3 is from linearity testing at the FSC in October of 2003. 
Another characteristic of superior resolution is the increased distance between the analyte spots and the dark pre-concentration zone at the bottom of the plate.

The efforts to determine the cause of the batch-to-batch resolution inconsistency led to a deeper understanding of various components of the TLC kit. Acceptance criteria for new batches of TLC plates were developed, along with a closer working relationship with the manufacturer. This relationship will prevent such anomalies from occurring in the future.

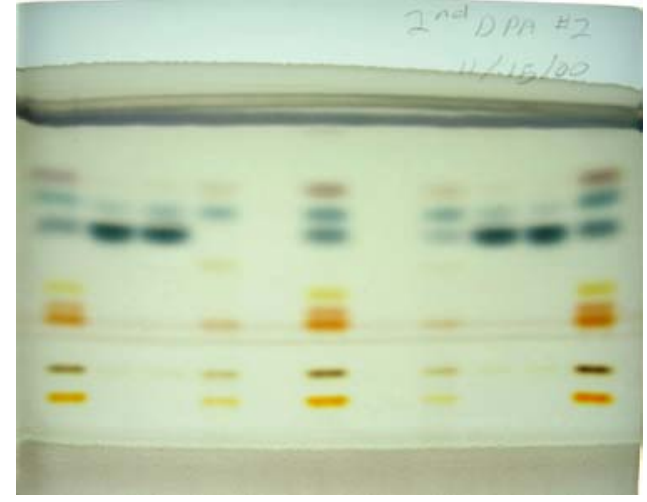

\#1-November 2000

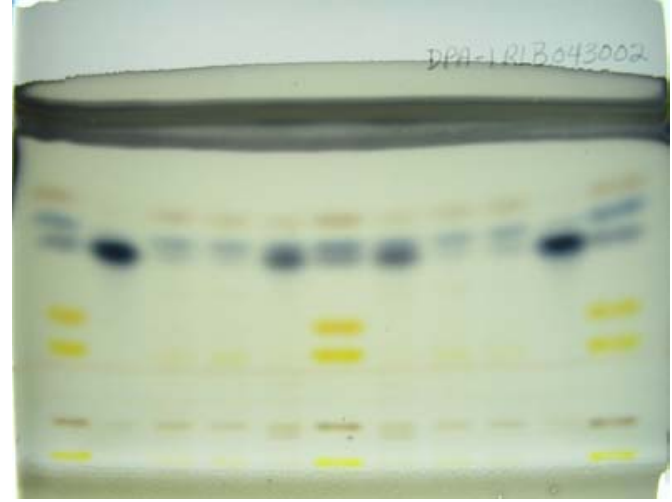

\#2-March 2002



\#3-October 2003

The normal TLC plate configuration is eleven parallel lanes, with standards in lanes 1, 6, and 11. However, because of erratic behavior at the edges of the plate, the strategy of sample layout or placement was addressed. The question was whether the two outside 
lanes should be eliminated, thereby reducing the number of samples run on each plate. Since it is not a frequent problem, and is less of an issue now that the plates are better understood, a software option was determined to be a better solution. Two equivalent programs are supplies with the kit computer, one that uses eleven lanes (standards in lanes 1, 6, and 11) and another for nine lanes (standards in lanes 2, 6, and 9). Additional software enhancements were also implemented as a result of knowledge gained investigating plate resolution.

The imaging system was also readdressed, and both the light panel and the digital camera were improved. The previous light panel was smaller and caused variations in lighting across the plate, therefore degrading the resulting digital image. The camera system was upgraded from a consumer product to professional grade, which will greatly increase consistency and product reliability in the future. The photo below shows the evolution of the imaging system during the project, with the generation time line increasing from left to right.

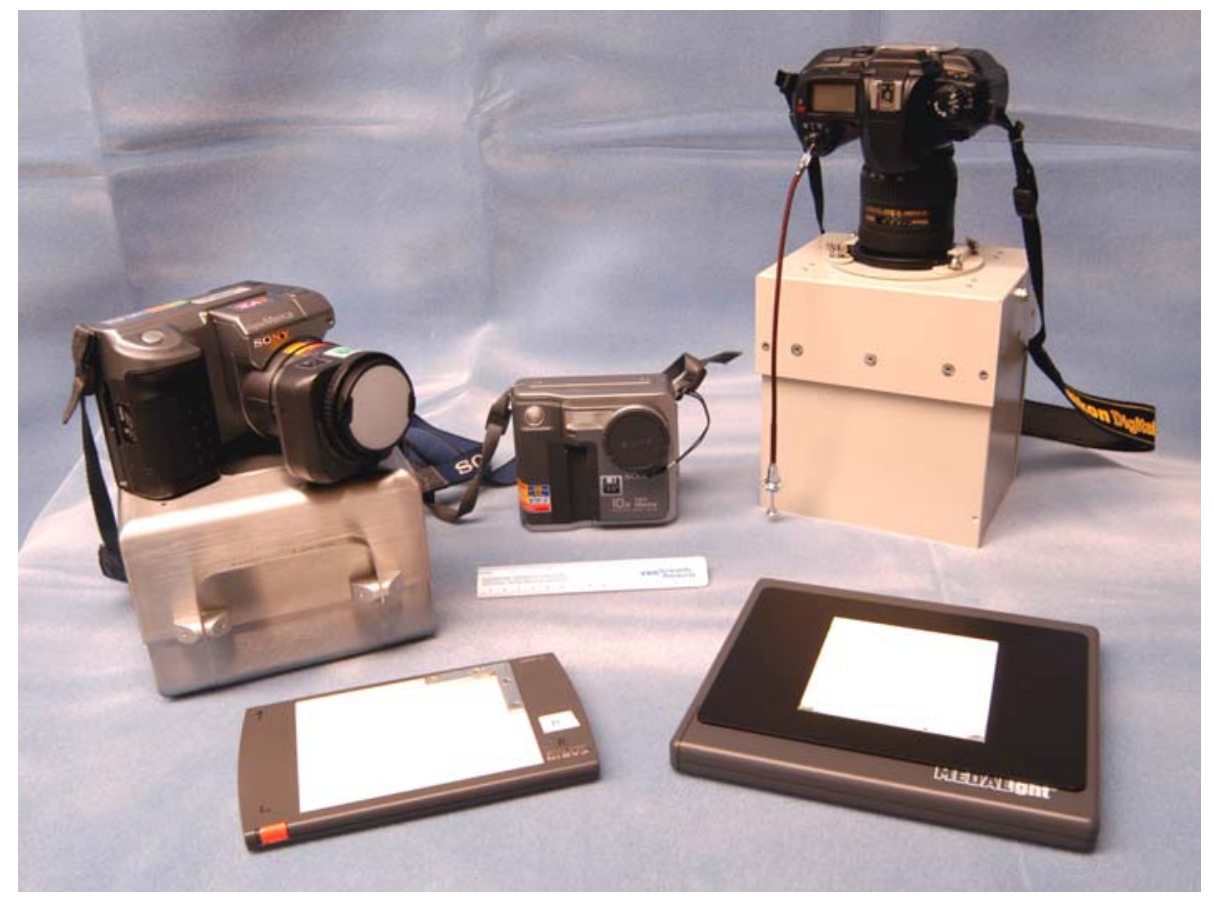

\section{Evolving Imaging System (Left to Right)}

As the project progressed, there were also issues related to the chemical reagent kit. The FSC worked very closely with Sigma-Aldrich Chemical, the parent company, and 
their subsidiary, Supelco, the chemical kit manufacturer, to develop a reliable, highquality kit. All chemicals were also transferred from the main kit and stored in a single case, similar to the one shown in the following photo. Steps were also taken to ensure chemical reliability and consistency in the field by including check standards for materials quality verification before any propellant lots are analyzed.

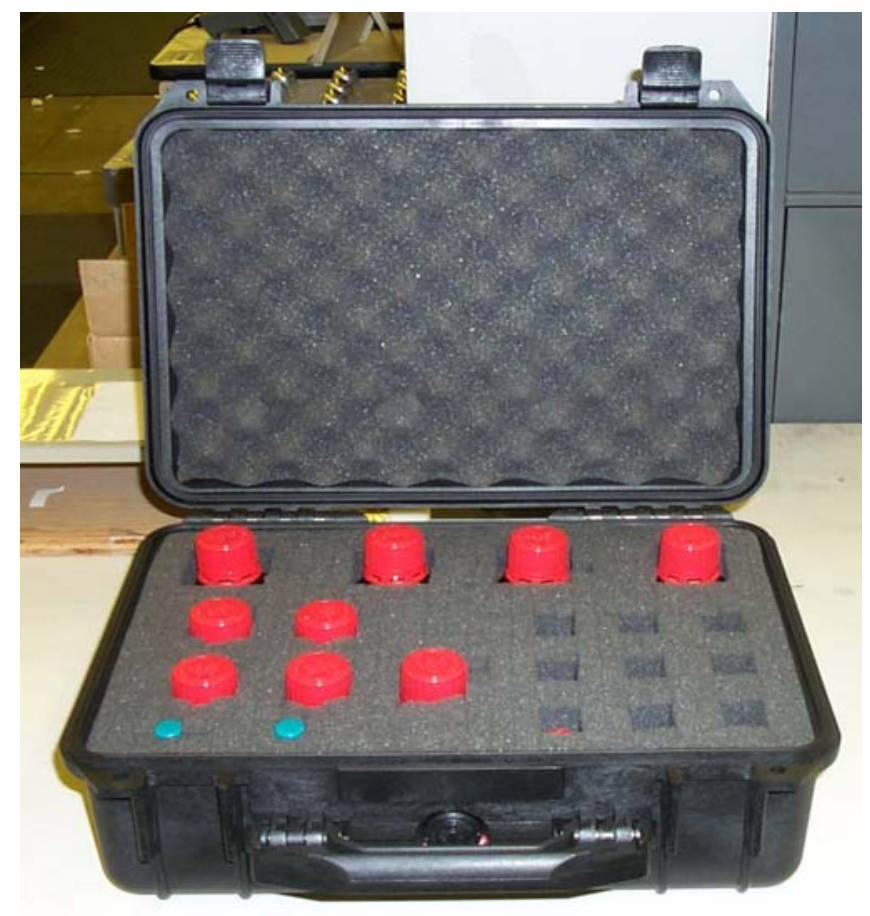

Chemical Kit

Propellant sampling issues were addressed as well. Due to the variety of shapes and sizes of propellants, new sampling methods were required. The photo below shows a novel propellant crusher, of our design, and cutters, that should allow the analyst to effectively sample most propellant configurations when used separately or combined. They are also easily cleaned between each use to prevent sample cross-contamination. 
UCRL 303212

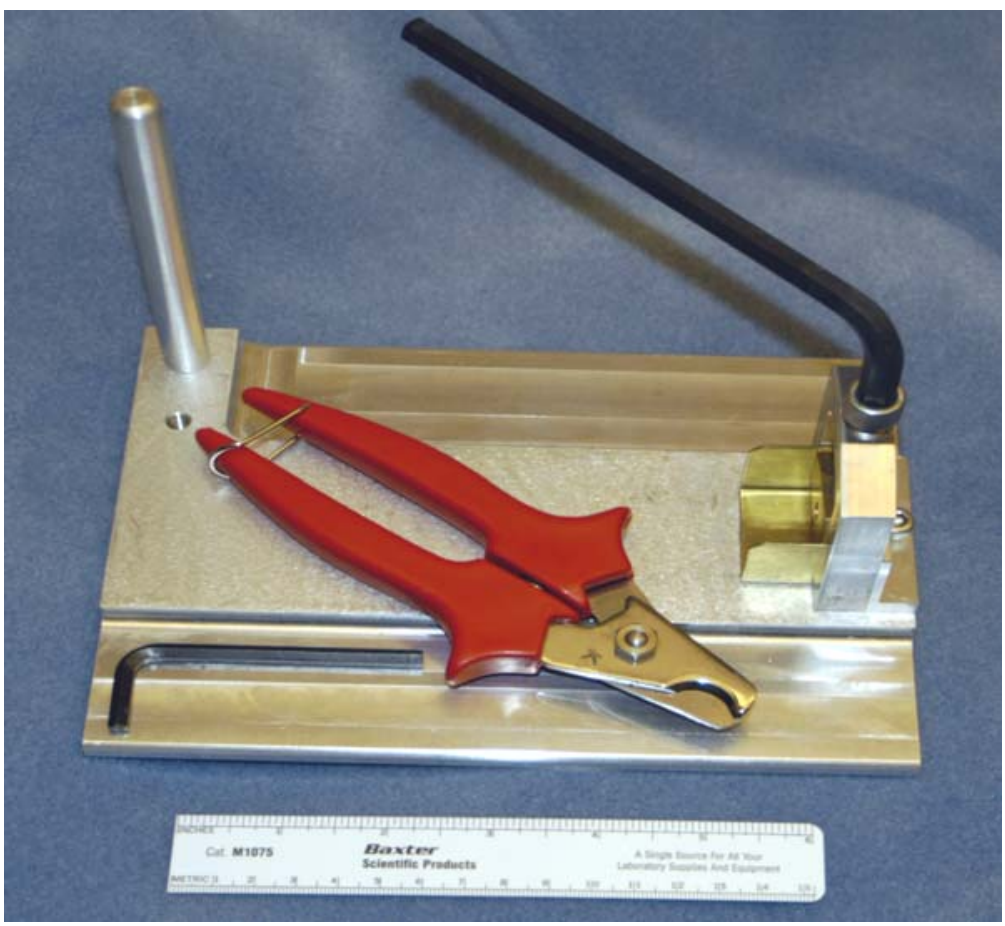

Sampling Equipment

\section{NOV 00 AND AUG 03 COMBINED RESULTS $\square \mathrm{MCAAP} \vee \mathrm{ARDEC}$}

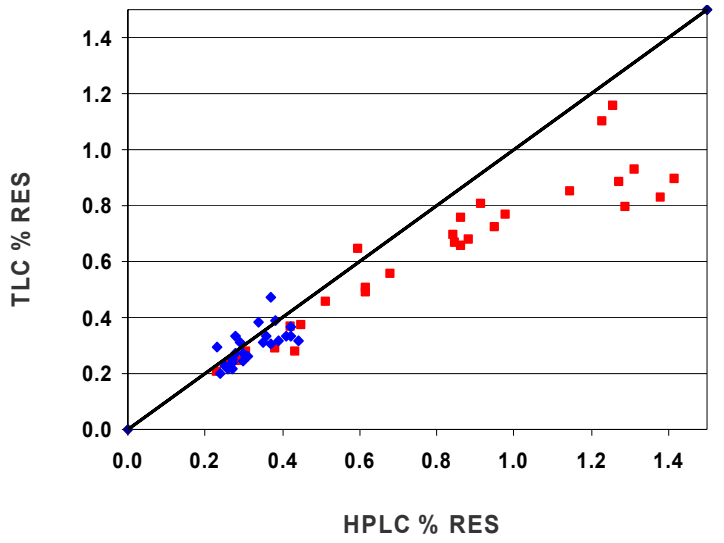

The displayed graph compares the results of two tests, one performed in November of 2000 at McAlester Army Ammunition Plant and the other at ARDEC/Geo-Centers during August of 2003. It shows the correlation between two methods, HPLC, the current "gold 
standard" for propellant analysis, and TLC. The November 2000 propellant data, comparing TLC with HPLC was considered to be very good by those involved at that time and therefore the TLC protocol was ready to proceed to validation. However, because of the problems discussed above, validation was not achieved. As is evident from the blue data points, from studies at Geo-Centers in August 2003, the agreement between TLC and HPLC is once again very good over a practical dynamic range. There is both good correlation and also now better understanding of the intricacies of the TLC system and potential difficulties.

Several areas that need investigation still remain for 2004. Some of those are onsite waste handling and the effects of humidity, temperature, and altitude on TLC separations. Training of field teams will be a main focus, with the goal to achieve validation during spring of 2004.

The attached funding chart summarizes project expenditures for 2003.

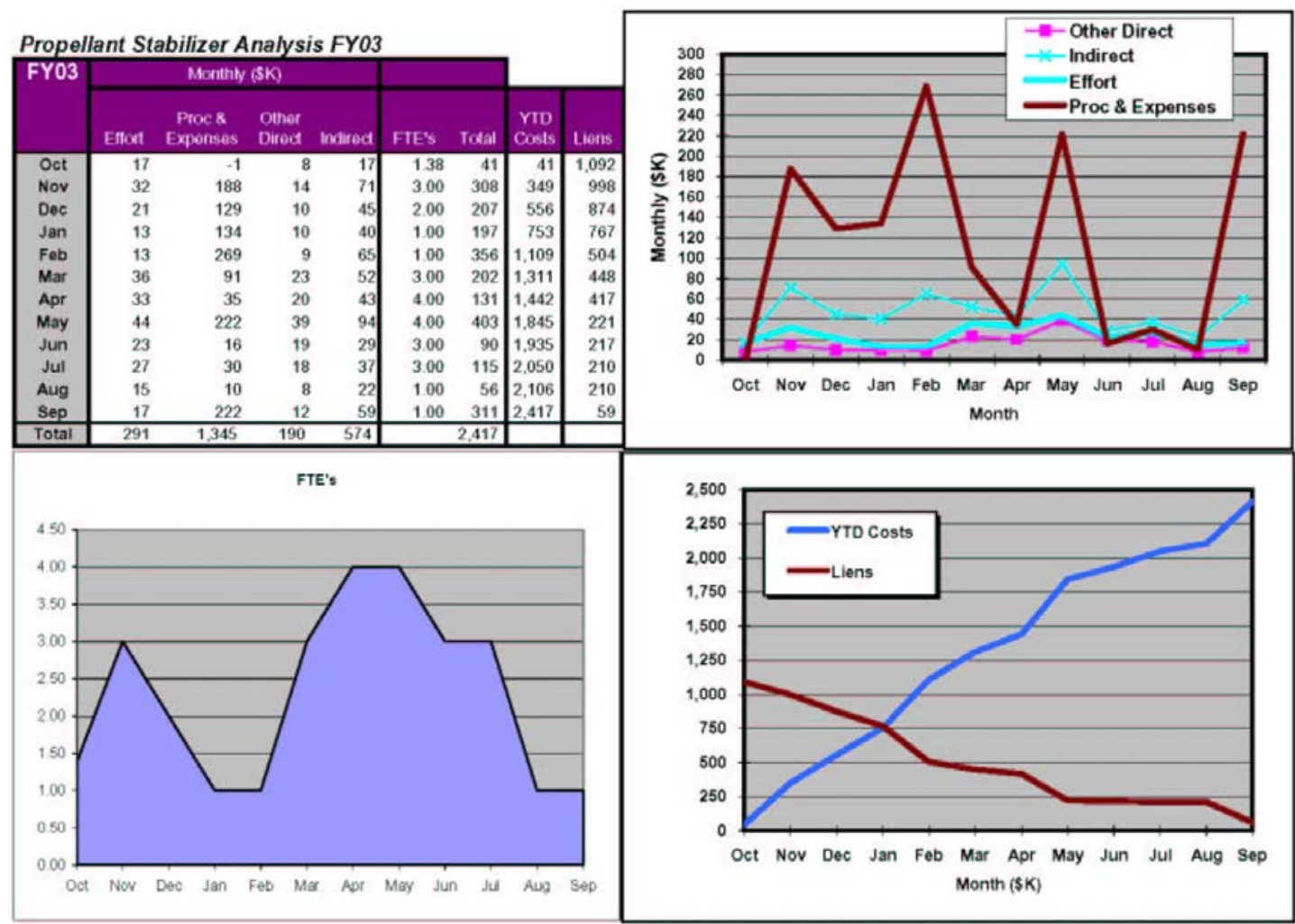

